Effects of palindromes on in vivo DNA replication and mutagenesis in bacteriophage ФX174 RF DNA

Uwe R.Müller*, Barbara K.Perkins and W.Lee Williams

Department of Microbiology and Immunology, East Carolina University School of Medicine, Greenville, NC 27858-4354, USA

Received June 16, 1988; Revised and Accepted October 17, 1988

ABSTRACT

Bacteriophage $\Phi \times 174$ mutants with insertions of palindromic DNA sequences are rapidly outgrown by competing wild-type phage (Müller \& Turnage, J. Mol. Biol. 189: 285). The basis for this defect was investigated and found to be due to an exclusion event early in the infectious cycle, in which phage genomes with palindromic inserts were preferentially excluded by wt phage. In addition, we have obtained further evidence for a palindrome induced genetic instability. Both defects are dependent on palindrome size and sequence, consistent with a model which involves formation of cruciforms, or cruciform-like structures. We propose that formation of unusual DNA secondary structures reduces the effectiveness of replicative form (RF) DNA to interact with limiting replication factors or membrane binding sites, possibly because of interaction with the host recombination system.

\title{
INTRODUCTION
}

The ability of certain DNA sequences to form unusual DNA secondary structures in vitro is well documented [for review see ref. 1], but evidence for their formation in vivo has come about only recently [2-4]. While our knowledge about Z-DNA formation and its potential role in gene regulation is rapidly expanding, much less is known about the biological significance of cruciforms, and even less information is available on the formation and function of slippage sites.

We have used the bacteriophage $\Phi \times 174$ system to study the formation, and putative structure-function relationship, of hairpins in the single-stranded genome, and cruciforms and slippage structures in the double-stranded RF DNA. To that end the palindromic sequence CCGGATCCGG (BamHI linker) or the sequence CCGAATTCGG (ECORI linker) were polymerized and then inserted into the unique PvuII site of the previously constructed 
बX174 mutant strain ins6 $[5,6]$. This resulted in palindromes of 18 to 48 base-pair (bp) size containing 1 to 4 directly repeated linker sequences. Thus, upon supercoiling the RF DNA, these sequences could theoretically be extruded either as cruciforms [7], or possibly as slippage structures [6]. In the singlestranded genomic DNA, and probably also in the polycistronic mRNAs, these sequences can also form hairpins [5]. Together with our collection of non-palindromic insertion and deletion mutants (Table 1) these mutants represent a powerful tool to test the effect of DNA and RNA structure on phage gene expression.

Any sequence changes that distinguish these mutants from the wild-type (wt) $\Phi \times 174$ strain are confined to the J-F

intercistronic region, which contains 27 non-essential nucleotides in $\Phi \mathrm{X} \mathrm{wt}$, but was enlarged to 144 nucleotides in strain ins6 [8,9]. A small, but statistically significant palindrome is located in the wt sequence, as well as in the J-F intercistronic region of the closely related bacteriophage G4 [10]. Modification of this palindrome either by deletion $[9,11]$, or by insertion of up to 164 additional nucleotides [9] resulted in genetically stable phage strains. Only when the insert size exceeded 164 nucleotides was genetic instability encountered [12]. However, the stability of mRNA [13], rate of protein synthesis [14], and even the eclipse kinetics [15] appeared to be affected by some of these sequence changes. Yet, a correlation between altered function and altered secondary structure, rather than sequence, remained speculative.

Insertion of palindromes into strain ins6 resulted in growth deficiencies [6; Müller et al., unpublished] and in some cases in a rapid deletion of the insert sequence $[5,6]$. Since these effects appeared to be dependent on the size and sequence of the palindrome, we have further investigated the underlying molecular mechanism.

MATERIALS AND METHODS

Bacterial and Phage strains

The bacteriophage $\Phi \times 174$ strains used in this work have all been described in detail before, and are summarized in Table 1. All strains carry the am3 mutation (an amber mutation in the $\mathbf{E}$ lysis gene), which results in the intracellular accumulation of 
Table 1. Phage strains used

\begin{tabular}{|c|c|c|c|c|c|c|c|}
\hline $\begin{array}{l}\text { बX174 } \\
\text { strain }\end{array}$ & & $\begin{array}{l}\text { Sequence } \\
\text { deleted }\end{array}$ & $\begin{array}{l}\text { modificat } \\
\text { inserted }\end{array}$ & $\begin{array}{l}\text { on in } \mathrm{J}-\mathrm{F} r \\
\text { palindrome }\end{array}$ & $\begin{array}{l}\text { gion } \\
\text { size \& }\end{array}$ & $\begin{array}{l}\text { in bp) } \\
\text { type }\end{array}$ & Ref. \\
\hline wt & & - & - & 16 & & wt & [28] \\
\hline del 3 & & 27 & - & - & & - & {$[9]$} \\
\hline insil & & - & 163 & - & & - & [9] \\
\hline$\overline{\text { ins } 6}$ & & - & 117 & - & & - & [9] \\
\hline ins 6210 & (E1) & - & $117+10$ & 18 & ECOR1 & monomer & [6] \\
\hline ins 6220 & (E2) & - & $117+20$ & 28 & ECOR1 & L dimer & [6] \\
\hline ins 6230 & (E3) & - & $117+30$ & 38 & ECOR1 & 1 trimer & [6] \\
\hline ins 6240 & (E4) & - & $117+40$ & 48 & ECORI & L tetramer & $=[6]$ \\
\hline ins 6430 & (B1) & - & $117+10$ & 18 & BamH1 & 1 monomer & [5] \\
\hline ins 6420 & (B2) & - & $117+20$ & 28 & BamH1 & I dimer & [5] \\
\hline ins 6463 & (B3) & - & $117+30$ & 38 & BamH 1 & 1 trimer & [5] \\
\hline$\overline{\text { ins } 6461}$ & (B4) & - & $117+40$ & 48 & BamHI & 1 tetramer & $=[5]$ \\
\hline
\end{tabular}

large numbers of progeny phage in E. coli C cells (non-permissive host). The presence of an amber suppressor in E. coli HF4714 (permissive host) restores $\mathrm{E}$ gene function.

\section{Media and Buffers}

TYE growth media contained $10 \mathrm{~g} / 1$ tryptone, $5 \mathrm{~g} / 1$ yeast extract, $5 \mathrm{~g} / \mathrm{l} \mathrm{NaCl}$, and $3 \mathrm{~g} / \mathrm{l} \mathrm{KCl}$. PDB buffer contained $1 \mathrm{M}$

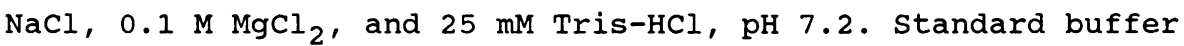
contained $50 \mathrm{mM}$ Tris- $\mathrm{HCl}, \mathrm{pH} 7.8,0.1 \mathrm{M} \mathrm{NaCl}$, and $10 \mathrm{mM}$ EDTA. All buffers and media were made $1 \mathrm{mM} \mathrm{CaCl}$ before phage infection. Enzymes

Restriction enzymes were purchased from International Biotechnologies, Inc., Bethesda Research Laboratories, New England Biolabs, or Boehringer-Mannheim. Restriction and storage buffers were either supplied by the manufacturer or prepared according to their specifications.

Production of phage stocks and RF DNA

Phage stocks were prepared by incubating well isolated single plaques with $1 \mathrm{ml}$ of a permissive host cell culture in early log phase $\left(10^{7} \mathrm{cells} / \mathrm{ml}\right)$ until lysis occurred. The phage titer was increased by successive additions of $5 \mathrm{ml}$ and then 15 $\mathrm{ml}$ permissive cells. This lysate was used to infect 20 to $30 \mathrm{ml}$ of non-permissive host cells $\left(2 * 10^{8} \mathrm{cells} / \mathrm{ml}\right)$, either for the production of high titer phage stocks or for production of $R F$ $D N A$. For phage production the infected cells were incubated for 2 $\mathrm{h}$, harvested and lyzed by treatment with lysozyme $(10 \mathrm{mg} / \mathrm{ml})$ and EDTA $(0.5 \mathrm{M})$, followed by freeze-thawing. For production of RF 
DNA the culture was mixed with chloramphenicol $(33 \mathrm{ug} / \mathrm{ml}$ final concentration) 5 to $7 \mathrm{~min}$ after phage infection, and incubated for $2 \mathrm{~h}$ before harvesting the cells. DNAs were then extracted by the miniprep procedure as described below, digested with restriction endonucleases, and analyzed by polyacrylamide gel electrophoresis (PAGE). Gels were stained with ethidium bromide and photographed with varying exposure times with polaroid type 55 film. Relative band intensities were determined by scanning negatives with an LKB Laser densitometer linked to an IBM 9000 mini-computer. Fragment sizes were determined using a computer program provided by J.R. Lowe [16].

\section{Small Scale preparation (Minipreps) of RF DNA}

Cell pellets from 10 to $30 \mathrm{ml}$ of infected cells were resuspended in 600 ul standard buffer in a conical $1.5 \mathrm{ml}$ centrifuge tube, mixed with 60 ul lysozyme $(10 \mathrm{mg} / \mathrm{ml}$ in standard buffer), and incubated at RT for $20 \mathrm{~min}$. Phenol $(0.5 \mathrm{ml}$ equilibrated with $0.1 \mathrm{M}$ Tris $-\mathrm{HCl}, \mathrm{pH} 8.0$ ) was added dropwise and tubes were spun for $20 \mathrm{~min}$ in a microfuge. The aqueous layer was carefully removed, extracted 2 more times with 300 ul phenol, mixed with 50 ul $3 \mathrm{M}$ sodium acetate, and $1 \mathrm{ml} 95 \%$ ethanol. After chilling in a dry-ice bath for $15 \mathrm{~min}$ precipitates were collected by centrifugation in the microfuge for 10-15 min. Supernatants were carefully removed and discarded. The pellets were washed with 300 ul cold ethanol, dried under vacuum, resuspended in 100 ul $0.3 \mathrm{M}$ sodium acetate, extracted twice with 200 ul ether, and precipitated once, followed by a wash, both with 300 ul 95\% ethanol in the dry-ice bath. Precipitates were collected by centrifugation, dried under vacuum, and resuspended in sterile $\mathrm{H}_{2} \mathrm{O}$.

\section{Determination of Phage Progeny in Mixedly Infected cells}

E. coli HF 4714 cells were grown in TYE to a density of $10^{8}$ cells/ml and harvested by centrifugation. After cells were washed with cold PDB they were resuspended in prewarmed PDB and incubated for $10 \mathrm{~min}$ at $37^{\circ} \mathrm{C}$. Aliquots were then infected with phage mixtures (m.o.i. of 10 per strain) and incubated for 15 additional min. Cells were pelleted, washed with cold PDB to remove unadsorbed phage, and resuspended in TYE. Aliquots were plated either directly onto agar plates to determine the cell 
survival rate $(<1 \%)$, or with permissive seed cells in soft agar to obtain isolated plaques. Plaques were transferred to $1 \mathrm{ml}$ permissive cell cultures for increases of phage titer and production of RF DNA as described above.

\section{RESULTS}

Competitive Synthesis of phage in one step Growth Experiments

We have tested the ability of phages with mutated J-F intercistronic regions to compete with wt phage in the same host cell. Aliquots of an E.coli C culture were infected simultaneously with $\Phi \mathrm{X}$ wt and one mutant strain (m.0.i. of 10 per phage strain). After $2 \mathrm{~h}$ incubation cells were lysed artificially, and the progeny phage was used to infect new cells. Chloramphenicol was added after $7 \mathrm{~min}$ and incubation continued for $2 \mathrm{hr}$. This allowed synthesis of double-stranded RF DNA, but prevented the shift to single-stranded DNA synthesis. The RF DNA was isolated by the miniprep procedure and analyzed for the distribution of phage genomes by digestion with HaeIII (Fig.1). The 8 largest fragments obtained after digestion of wt RF DNA with HaeIII are shown in the first and last lane and are labeled on the right side of Fig.1. The presence of fragments 5 and 8 is characteristic for wt DNA, since the HaeIII site between these two fragments has been destroyed in the construction of strain ins 6 and ins11. The resulting fusion fragments are devoid of a HaeIII site and migrate above fragment 4 (labeled 5-x-8). In strain del 3 a deletion of 27 nucleotides has removed the HaeIII site between fragments 5 and 8 and the resulting fusion fragment migrates below fragment 4 . Insertion of a single BamHI or ECoRI linker into the pvuII site of strain ins6 results in a $10 \mathrm{bp}$ size increase of the 5-x-8 fusion fragment. Insertion of 2 or more linkers, however, results in the generation of HaeIII sites between two linkers. Consequently, in those cases HaeIII cleaves the 5-x-8 fusion fragment into two new sub-fragments, labeled 5$y-8$ and 5-z-8, respectively.

The intensity of mutant specific fragments was compared to that of wt specific fragments (or to fragments contributed by both genomes) to estimate the ratio of wt to mutant RF DNA present after one complete life cycle followed by an incomplete 


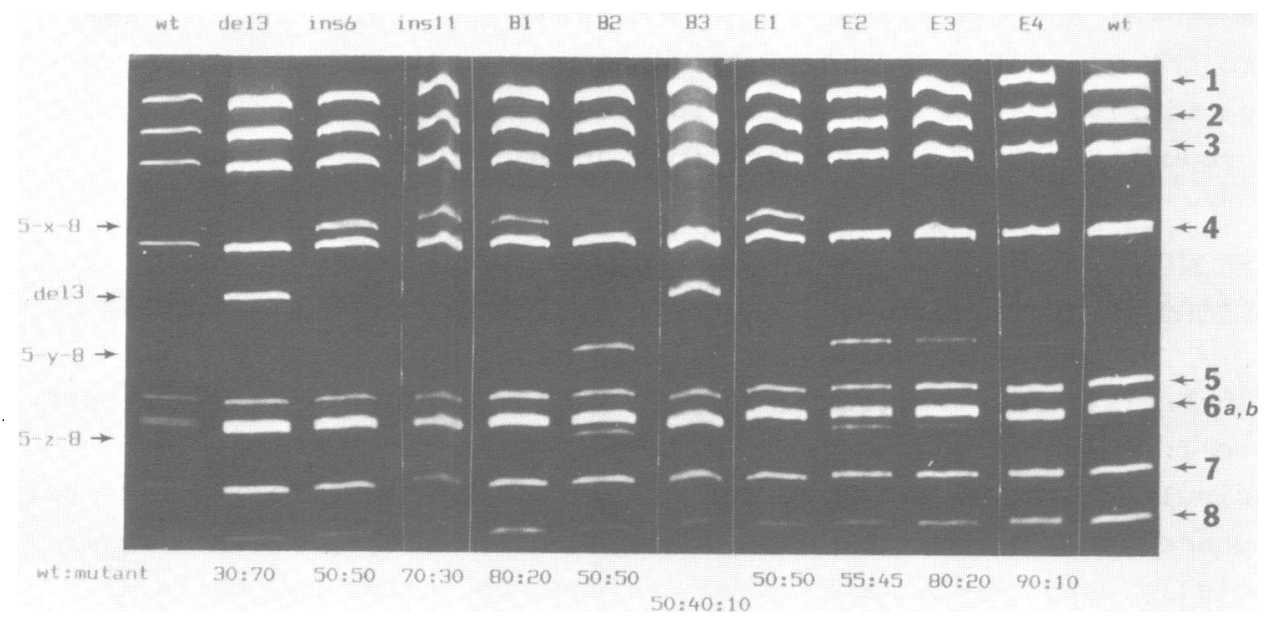

Figure 1. Competitive growth and RF DNA synthesis. Aliquots $(12 \mathrm{ml})$ of an E. coli C culture $\left(4 * 10^{7} \mathrm{cell} / \mathrm{s} / \mathrm{ml}\right)$ were washed with $12 \mathrm{ml}$ cold $\mathrm{PDB}$, and after pelleting the cells, were resuspended in warm $\mathrm{PDB}$. After shaking at $37^{\circ} \mathrm{C}$ for $10 \mathrm{~min}$ cultures were made $1 \mathrm{mM} \mathrm{CaCl}{ }_{2}$ and mixed with phage (wt and one mutant strain; each at an m.o.i. of 10). After shaking for $15 \mathrm{~min}$ at $37^{\circ} \mathrm{C}$ infected cells were separated from unadsorbed phage by centrifugation, washed with cold PDB once, and resuspended in 12 $m l$ TYE. Titer of surviving cells suggested an infection rate of $99 \%$. After shaking for $2 \mathrm{~h}$ at $37^{\circ} \mathrm{C}$ cells were harvested by centrifugation, and phages released by treatment with lysozyme, EDTA, and freeze-thawing. Phage titers were determined and used to infect $10 \mathrm{ml}$ cultures of $\mathrm{E}_{\text {. }}$ coli $\mathrm{C}$ (m.o.i. of 5). Chloramphenicol was added after 7 to $8 \mathrm{~min}$ and cells were harvested $2 \mathrm{~h}$ later. RF DNA was prepared by the miniprep procedure, digested with HaeIII, run on a $5 \%$ PAGE gel, and band intensities quantitated as described in the Method section. A digest of purified wt RF DNA was run in the first lane $(0.5 \mathrm{ug}$ DNA) and last lane (2 ug DNA) respectively. The phage strain used in the co-infection with wt is indicated above each lane. The approximate ratio of wt to mutant DNA was calculated from the intensity ratio of wt specific HaeIII fragments 5 and 8 (indicated on right side of gel) to one of the mutant specific fragments (indicated on the left side of gel), and is indicated below each lane. In case of strain $B 3$ the ratio of $50: 40: 10$ refers to wt: del3:B3.

cycle (up to RF DNA synthesis). The 50:50 ratio found in the wt/ins 6 mutant mixture suggests that insertion of $117 \mathrm{bp}$ into the $J-F$ region did not result in a detectable phage growth deficiency. Insertion of $163 \mathrm{bp}$ (insl1), however, caused a detectable decrease in growth efficiency, suggesting a possible effect of genome size. A much stronger effect was observed by 
several of the palindromic linker insertions into strain ins6, even though all of these mutants had a smaller genome size than insll. clearly, a substantial reduction in growth efficiency was observed in the ECORI linker mutants concomitant with the increase in palindrome size. The effect of BamHI linker palindromes was at least as significant. The trimeric linker insert is genetically unstable and frequently causes the deletion of all non-essential nucleotides from the J-F region [6], which resulted in the appearance of a deletion mutant very similar (and most likely identical) to strain del3. Both this strain, as well as $\Phi \mathrm{X}$ wt, outgrew the BamHI trimer insert strain (B3) nearly completely. The B4 containing strain was not used in this experiment since we have never been able to grow it in mixed infections with wt phage without complete conversion to a deletion mutant (see below).

\section{Competitive synthesis of RF DNA}

The preceding experiment revealed a reduced ability of some mutants to compete with wt phage in mixed infections. We have searched for what appears to be a palindrome induced defect early in the infection cycle by infecting cells at high multiplicity with mutant phage in the presence or absence of wt phage. Chloramphenicol was added $5 \mathrm{~min}$ past infection and incubation continued for $2 \mathrm{~h}$ to accumulate RF DNA, which was purified by the miniprep procedure and digested with HaeIII. The ratio of wt to mutant DNA was determined as in the foregoing experiment. Fig. 2 reveals that the above observed palindrome mediated growth deficiency is already visible at this stage in the infectious cycle. While the effect of the monomer and dimer linkers is at best small, both the ECORI and the BamHI trimer bearing strain were unable to produce the same amount of RF DNA as $\Phi \times 174$ wt and contributed only approximately $10 \%$ to the total amount of RF DNA under these conditions. In case of the BamHI trimer both RF DNA (+ and - wt phage) preparations contained a high concentration of a deletion mutant, which appears to be identical to del $3[6,12]$. since the ins 6463 stock used for these experiments was at least $95 \%$ pure, most or all of this DNA must have been generated from the B3 strain RF DNA during this replication cycle. For the EcoRI tetramer bearing strain comparison with the wt specific bands in 


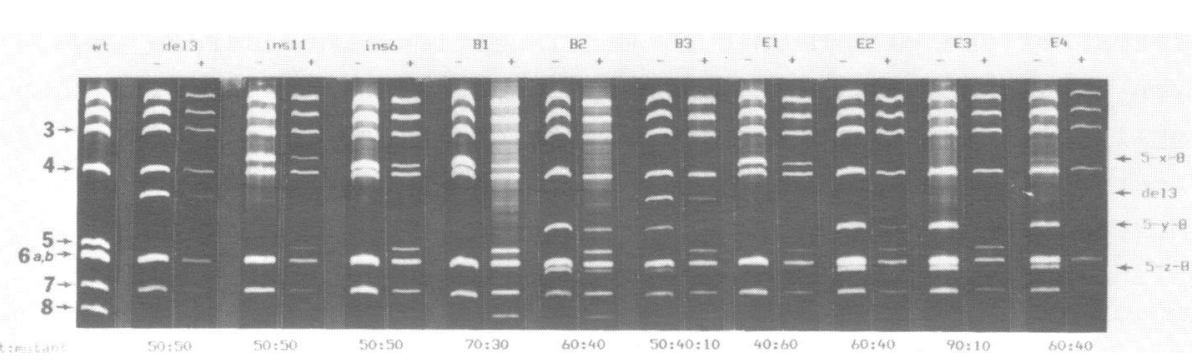

Figure 2. Competitive synthesis of RF DNA.

Aliquots $(30 \mathrm{ml})$ of an E. coli C cell culture were infected with either a single phage type (m.o.i. of 20), or with a mixture of wt and one mutant phage (m.o.i. of 10 per strain).

Chloramphenicol was added $5 \mathrm{~min}$ later and RF DNA was extracted by the miniprep procedure after $2 \mathrm{~h}$ incubation. Equal aliquots of each miniprep were digested and the bands quantitated as described in Fig.1. The first lane contains the wt control culture (m.o.i. of 20$)$. The other lanes are labeled with the strain type used alone (-) or in mixture with wt $(+)$. All other labels are as in Fig. 1.

Fig.2 suggests only a slight disadvantage in RF DNA synthesis in this experiment.

Since approximately equal aliquots of the miniprep RF DNA extracts were digested and applied to the gel, it appears that in the absence of competition the amount of RF DNA synthesized for each phage is similar under these experimental conditions. However, for the mixed infections the results suggest that at least some of the palindromes (i.e. the trimers) seriously affect the phage's ability to replicate RF DNA when in competition with wt and/or deletion mutants, that have spontaneously deleted the palindrome.

\section{Effect of Palindromes on Phage Exclusion}

Salivar and Sinsheimer [17] have reported that in prestarved host cells only an average of 1.6 बX RF DNA molecules can participate in replication, apparently due to the limiting availability of functional replication sites or limiting concentrations of an essential replication factor. Thus, simultaneous infection of a cell with 2 types of phages should lead to exclusion of one phage type in at least half of all infective centers. The results of Borrias et al. [18] are consistent with such a hypothetical exclusion process at the level of replication. 
Table 2. Effect of palindromic sequences on replication exclusion and genetic stability

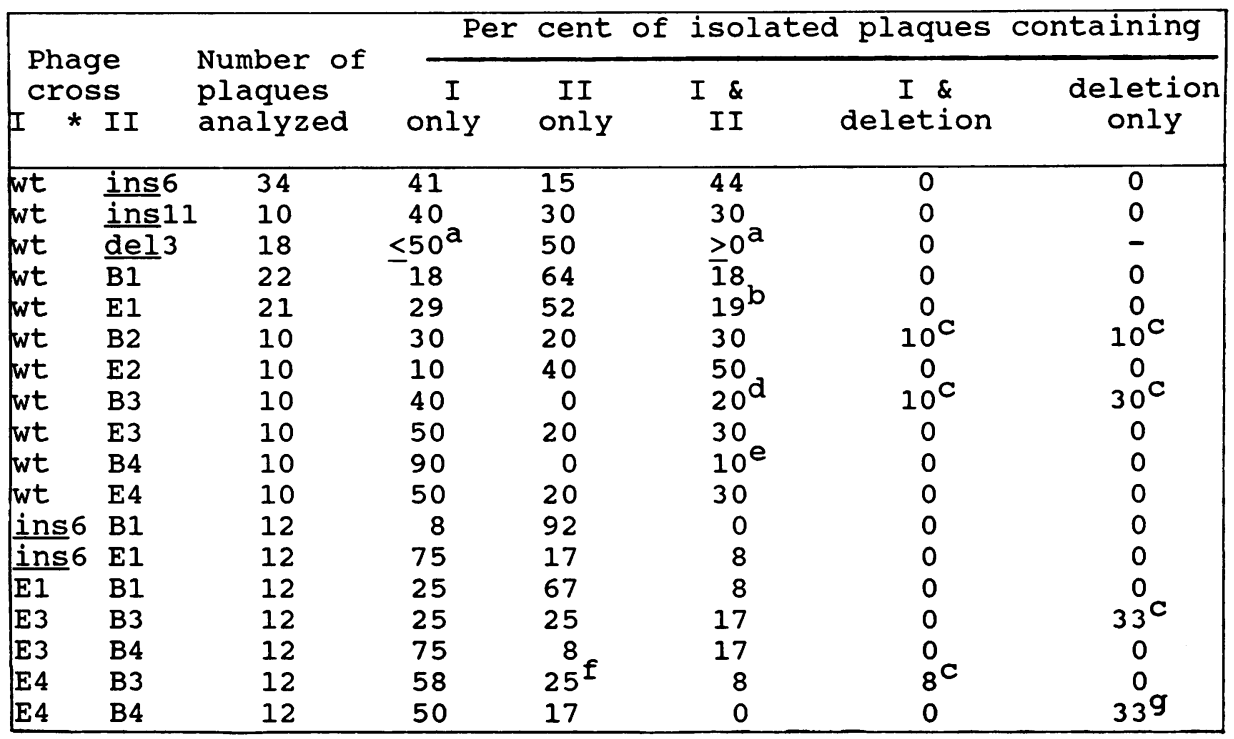

a) In 8 of these digests the presence of some del 3 in addition to wt could not be ruled out.

b) Out of 4 mixtures 3 contained in addition a strain similar to E2.

c) All deletions were similar to del3.

d) Both mixtures contained between $40 \%$ and $60 \%$ B3, and small amounts of del3.

e) This mixture contained only a trace of B4 (5 to 10\%).

f) Judged only by presence of BamHI site but not by sizing of palindrome.

g) In each case the deletions had retained one linker unit.

In light of our preceding results we have tested whether the presence of large palindromic sequences might affect the ability of an RF DNA to compete with the wt DNA for a replication site or a limiting replication factor. Permissive cells were washed and then incubated in buffer to induce starving conditions. Cells were then simultaneously infected with 2 different phage strains at a multiplicity of infection (m.o.i.) of 10 per strain, incubated for $15 \mathrm{~min}$, and washed to remove unadsorbed phage. These original infective centers were plated with permissive seed cells. Individual well isolated plaques were picked and analyzed for the composition of progeny phages by propagation of phage and production of RF DNA in small cultures, and digestion of the 
resulting RF DNA mixtures with HaeIII and/or HhaI, similar to Figs. 1 and 2. When appropriate, these RF mixtures were also tested for the presence or absence of restriction sites (PvuII, EcoRI, BamHI) that are characteristic for a specific strain. The results of over 240 such experiments are summarized in Table 2 . Doubly infecting permissive cells with wt phage and one of the J-F mutants resulted in plaques containing both types of phages in only $30 \%$ of all cases. More frequently, one of these strains was eliminated or at least reduced to less than $5 \%$ of the total phage progeny in a single plaque (the limit of our detection method). This clearly demonstrates that the predicted process of phage exclusion exists. Furthermore the first three sets of experiments suggest that modification of the J-F intercistronic region either by the insertion (ins6 and ins11) or deletion of nucleotides (del 3 ) does not affect this process, and both wt and mutants appear to have a roughly equal chance of being eliminated. This is not true, however, for mutants with palindromic insertions. In competition with wt phage the chances for the BamHI linker containing strains to be the sole survivor in a plaque were roughly $64 \%$ for B1, $20 \%$ for B2, and $0 \%$ for B3 and B4. For the EcoRI linker strains the chances were $52 \%$ for E1, $40 \%$ for E2, and $20 \%$ for E3 and E4. These data suggest an inverse correlation between palindrome size and the ability of the corresponding phage to compete with wt in this exclusion process. The sequence of the palindrome, i.e. its $G, C$ content, also seems to play a role. Both, the G,C content and the length of the palindromic region are important parameters for the physical stability of a hairpin in the single-stranded DNA or for formation of a cruciform in the double stranded DNA. These data are therefore consistent with a model in which the increased physical stability of a hairpin-like secondary structure leads to a decreased chance of the genome to compete for a limiting site or factor in the replication process. This model receives further support from the results of experiments in which the linker bearing mutants were competing against each other (Table 2). However, the presence of a small G,C rich structure in the J-F region appears to be of some advantage, since the B1 strain seems to outcompete its parent ins6, the wt phage, and even its counterpart E1 in this assay. 
Table 3. Effect of palindromes on competitive RF DNA replication

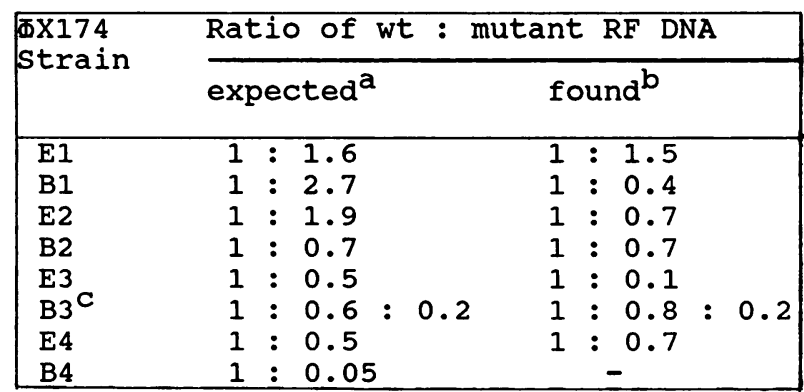

a) Calculated from the wt to mutant distribution in Table 2 .

b) Data from Fig.2.

c) Expected and found ratios are wt:del3:B3

Based on the data in Table 2 we should be able to roughly predict the ratio of wt to mutant RF DNA synthesized in doubly infected host cells. For this purpose we assume that those plaques producing two types of phages (columns I\& II and I\& deletion) produced those in equal numbers (which was not always true). Considering that the number of plaques analyzed in Table 2 is relatively low for most phage mixtures, Table 3 shows a fairly good agreement between this theoretical ratio and the ratio determined in the experiment of Fig.2. With the exception of strains B1 and E2, the inability of palindrome containing mutants to compete against wt phage for a limiting replication site (or factor) appears to be a satisfactory explanation for their inability to produce the same amount of RF DNA as wt under those conditions.

\section{Effect of Palindrome size and sequence on Deletion Type and} Frequency

We have previously reported a correlation between the sequence, as well as the size, and the genetic stability of palindromes that were inserted into the $\Phi \times 174$ genome [6]. When infecting host cells with phage, or transfecting with RF DNA, using either strains $B 3$ or $B 4$, we noted repeatedly the rapid appearance of specific deletion mutants. Strain B4 deleted either 2 or 3 of its 4 linker units, resulting in strain B2 or B1. Strain B3 deleted either 2 of its 3 linker units, resulting in strain $B 1$, or all non-essential nucleotides from the $J-F$ 
intercistronic region (judged by restriction mapping), resulting in a strain similar (or identical) to del3. Much less frequently strain E4 also gave rise to deletion mutants, similar to B4, but all other strains appeared to be absolutely stable. Thus, two different deletion processes were encountered. The first type removed specifically one or more linker units, but always left at least a perfect $18 \mathrm{bp}$ palindrome behind, leading us to suggest formation of off-set cruciforms through DNA slippage as the underlying mechanism. The second deletion process was equally specific but remains unexplained [6].

Table 2 reveals that both types of deletions were obtained in the mixed infection experiments as well, and since all experiments were performed under identical conditions, they allow some estimate of the frequency of deletion formation. To improve the accuracy of that estimate we have repeated the mixed infection experiments using only one type of phage, i.e. cells were infected with a single phage at an m.o.i. of 20 , spread onto plates and individual plaques were analyzed (Table 4). We interpret the data in Table 2 and Table 4 with the following assumptions. With a burst size of approximately 100 progeny phages per infected cell it is unlikely that a cell neighboring an infective center would be infected with only one phage. Due to the above described exclusion mechanism a deletion event in the second phage generation, i.e. in one of the cells neighboring the original infective center, would have little chance to establish itself. Hence, a mutation arising in a plaque, and emerging as the only type of phage in that plaque, must have been generated in the initial infective center. That is most likely also true for deletion mutants that were found in plaques containing more than one type of phage. We argue that such mixtures originated from the original infective center in which two genomes were replicated, one of which was mutated. This hypothesis is further supported by the finding of plaques containing only wt phage and a single deletion mutant instead of the parental palindrome containing strain.

Both Table 2 and Table 4 clearly suggest that the BamHI linker palindromes are much more unstable than the EcoRI linkers. In fact, not a single deletion event was observed in over 40 
Table 4. Effect of palindromic sequences on genetic stability

\begin{tabular}{|c|c|c|c|c|}
\hline \multirow{2}{*}{$\begin{array}{l}\text { Phage } \\
\text { selfings } \\
I * I\end{array}$} & \multirow{2}{*}{$\begin{array}{l}\text { Number of } \\
\text { plaques } \\
\text { analyzed }\end{array}$} & \multicolumn{2}{|c|}{ Per cent of plaques } & containing \\
\hline & & $\begin{array}{c}I \\
\text { only }\end{array}$ & $\begin{array}{c}\text { I \& } \\
\text { deletion }\end{array}$ & $\begin{array}{c}\text { deletion } \\
\text { only }\end{array}$ \\
\hline E1 & 22 & 100 & 0 & 0 \\
\hline B1 & 26 & 100 & 0 & 0 \\
\hline E2 & 20 & 100 & 0 & 0 \\
\hline $\mathrm{B} 2$ & 20 & 100 & 0 & 0 \\
\hline E3 & 16 & 100 & 0 & 0 \\
\hline B3 & 10 & 80 & 0 & $20 a$ \\
\hline E4 & 13 & 85 & $8^{b}$ & $8^{b}$ \\
\hline B4 & 14 & 14 & $7^{C}$ & $79^{d}$ \\
\hline
\end{tabular}

a) All deletions appeared to be identical to del3.

b) $91 \mathrm{bp}$ were deleted from the J-F region.

c) All deletions appeared to be identical to B1.

d) Nine of 11 deletions appeared identical to $\mathrm{Bl}$ and 2 appeared identical to B2.

plaques that originated from cells in which the $\mathrm{El}$ genome was replicated (combination of Table $2 \& 4$ ), which means that the probability of deleting the EcoRI monomer is less than $3 \%$. Similarly, the E2 and E3 genomes appeared stable and if a deletion is possible, that frequency must be also below $3 \%$ or $5 \%$ respectively. In 2 out of 18 cases (we ignore plaques containing only wt phages) E4 gave rise to a deletion, placing that frequency at roughly 10\%. However, in both these cases restriction analysis revealed that the deletion involved approximately $91 \mathrm{bp}$, representing possibly a third class of deletion events specific only to E4. A very similar (and possibly identical) type of deletion was obtained in a different experiment using strain E4, which supports this notion (not shown).

For the BamHI linker palindromes we have calculated the deletion frequencies based on Table 2 and 4 to be less than $2 \%$ for B1 and B2 (no deletions observed), 38\% for B3, and $86 \%$ for B4. For the latter two the types and frequencies of deletions obtained were expected (Figs. $1 \& 2$ ) and consistent with all previous data $[5,6]$.

Together these data strongly support our previous conclusion that the sequence as well as the length of a palindrome have a profound effect on its genetic stability. These two parameters 
have different effects not only on the frequency, but also on the type of deletions that are generated. In addition we conclude that the palindromes are deleted very early in the infectious cycle, certainly before single-stranded DNA synthesis begins. Effect of Palindromes on Recombination

We have previously obtained some evidence that transfection of host cells with large palindrome containing RF DNAs may result in high frequency recombination events at the palindromic region, either increasing the size of a palindrome, or combining the ECoRI linker and BamHI linkers from two different genomes into one [6]. The experiments described above were initiated to further test this possibility, but the results in Table 2 and 4 clearly suggest that the $\Phi \times 174$ system is inappropriate for such studies. First, the exclusion of all but a few invading genomes from replication severely reduces the chances for a recombinant genome in doubly infected cells to be replicated. In addition, the very rapid excision of large palindromes from the genome would make any recombination event that generates a palindrome with more than 4 ECORI linkers or more than 2 BamHI linkers very unstable, further reducing its chances to be detected. Nevertheless, we have obtained additional evidence for the recombinatory activity of these palindromes. In the cross of wt with El (Table 2) only 4 out of the 22 plaques tested contained a mixture of both phages. Three of these mixtures contained in addition to the input phages a third strain that appeared identical to $\mathrm{E} 2$ in restriction digests, possibly arising by duplication of the ECORI linker.

An even more unusual recombination event was observed when the E4 RF DNA shown in Fig. 2 was further analyzed. While the HaeIII digest gave the expected fragments, a HhaI digest revealed that one of the fragments had increased in size and a neighboring fragment had decreased in size by approximately the same number of bp, suggesting that the HhaI site was moved by approximately $14 \mathrm{bp} \mathrm{(not} \mathrm{shown).}$

\section{DISCUSSION}

We have observed that insertion of more than 2 BamHI or ECORI linker units into the pvuII site of $\Phi \times 174$ strain ins6 
renders the phage less competitive when growing in the presence of the wt phage. This defect appears to be at the level of DNA replication. There are basically two mechanisms that could explain these data. The first possibility would be a reduced rate of RF DNA synthesis, which might result in relatively less mutant RF DNA under conditions where the available time, precursor pool, or energy is limiting. The other possibility is the inability of mutant DNA to initiate replication as efficiently as wt DNA.

Our data suggest that in the absence of competition, RF DNA synthesis in mutant infected cells is similar to that in wt infected cells (Fig.2 and unpublished results). When competing against wt, however, less mutant specific RF DNA can be extracted from the cell culture when simultaneously infected with wt phage and a mutant containing a trimeric or tetrameric linker insert. Since this was not true for the control strain insl1, which contains 164 additional bp in the J-F region compared to the 157 additional bp in the largest linker insert strains, this effect appears to be palindrome specific.

The reason for this seems to be an inability of these mutants to initiate RF DNA replication as quickly or as efficiently as wt phage. The underlying mechanism is likely to be linked directly or indirectly to a phage exclusion mechanism that was proposed to exist at the level of DNA replication by other laboratories $[17,18]$. Our data strongly support that such a mechanism exists, and suggest further that genomes with large palindromes are excluded more frequently. Moreover, not only the size but also the sequence of the palindrome exerts an effect, consistent with a model in which formation of a stable hairpin, cruciform, or slippage structure interferes with replication.

since replication of RF DNA in the infected cell is preceded by conversion of the incoming single-stranded genome to a doublestranded molecule and subsequent expression of phage gene $A$ [for review see ref 19], there are several ways for a palindromic sequence to interfere with the regulated process. First, formation of a stable hairpin in the single-stranded DNA, which we have demonstrated in vitro [5], may impede the synthesis of the complementary strand. Second, formation of a stable secondary structure in the double-stranded template, or formation of a 
hairpin in the polycistronic mRNA, may somehow affect the expression or action of gene A. Finally, extrusion of the palindrome into a cruciform or slippage structure could interfere with the replication machinery either directly, or indirectly by reducing the torsional stress. Whatever the mechanism, it has to explain why replication of genomes with large palindromes is specifically affected in the presence of genomes without these sequences/structures.

Formation of a non-B secondary structure in the RF DNA is the most attractive model, because it can be used to explain not only the phage exclusion, but also the observed palindrome induced deletion and recombination process. Here too the strong dependence on palindrome size and sequence suggests a model in which the stability of a cruciform-type structure catalyzes a deletion process, which may actually start as a recombination event. We have previously proposed, that large palindromes are substrates for increased recombination [6], generating additional copies of the palindromic sequence, as we have observed for the El strain (Table 2). Doubling of the trimer or tetramer linker palindromes would result in palindromic sequences which are so unstable, that they are deleted before the phage strain can be isolated $[5,6]$.

Based on all our data we propose the following model. When the single-stranded genome enters the host cell, a complementary strand is made and the parental RF DNA is supercoiled [20]. This allows formation of a variety of unique secondary structures, but most of these are short-lived and unstable [7]. Insertion of large palindromes generates potential sinks of supercoil energy, with which the normal supercoil-dependent secondary structures can not compete. Once formed, these structures resemble Holliday junctions, and become targets for resolution [21,22] and recombination $[6,23,24]$. Possibly because of the unusual conformation of the DNA, and/or the absence of a replication fork, this results generally in excision of part or all of the palindromic sequence, including even some of the neighboring nonessential nucleotides $[6,25,26]$. Possibly because of these activities, or an altered conformation of the parental RF DNA due to palindrome extrusion, this molecule is not as good a substrate 
as the wt RF DNA for limiting replication factors or replication sites on the membrane. This results in exclusion of the mutant genome from replication in the presence, but not the absence of competing wt DNA.

An alternate explanation may be that extrusion of the inserted palindromes decreases the superhelical density sufficiently to prohibit extrusion of the smaller naturally occuring palindrome in the $\mathrm{H}-\mathrm{A}$ intercistronic region of the $\mathbf{} \mathrm{X} 174$ genome. When cloned into a multicopy plasmid, this sequence was shown by Baas et al. to bind to a limiting membrane site or replication factor, and inhibit replication of infecting phage DNA [27].

Experiments are in progress to investigate whether the effects of these sequences are due to their palindromic or direct repeat nature, and whether or not they are limited to the $\Phi \times 174$ system.

\section{ACKNOWLEDGEMENTS}

This work was supported in part by National Institutes of Health Grant GM31993 to U.R.M.

* To whom correspondence should be addressed

\section{REFERENCES}

1. Wells, R.D. and Harvey, S.C. (eds), (1987) Unusual DNA Structures, Springer Verlag Publishers, New York, NY.

2. Hsu, M.T. (1985) Virology 143,617-621.

3. Jaworski, A., Hsieh, W.-T., Blaho, J.A., Larson, J.E. and Wells, R.D. (1987) Science 238,773-777.

4. Panayotatos, N. and Fontaine, A. (1987) J. Biol. Chem. 262,11364-11368.

5. Müller, U.R. and Turnage, M.A. (1986) J. Mol. Biol. 189,285292.

6. Williams, W.L. and Müller, U.R. (1987) J. Mol. Biol. 196,743755.

7. Müller, U.R. and wilson, C.L. (1987) J. Biol. Chem. 262,37303738 .

8. Müller, U.R. and Wells, R.D. (1980) J. Mol. Biol. 141,1-24.

9. Müller, U.R. and Wells, R.D. (1980) J. Mol. Biol. 141,25-4l.

10. Tao-shih, H. and Wang, J.C. (1975) Biochem. 14,527-535.

11. Müller, U.R. (1983) J'. Virology 48,170-179.

12. Russell, P.W. and Müller, U.R. (1984) J. Virology 52,822-827.

13. Hayashi, M., Hayashi, M.N. and Müller, U.R. (1983) J.' Virology $48,186-196$. 
14. Romantschuk, M.I. and Müller, U.R. (1983) J. Virology 48,180185.

15. Incardona, N.L. and Müller, U.R. (1985) J. Mol. Biol. $181,479-486$.

16. Lowe, J.R. (1986) Federation Proceedings 45,1852.

17. Salivar W.O. and Sinsheimer, R.L. (1969) J. Mol. Biol. 41,3965 .

18. Borrias, W.E., Van De Pol, J.H., Van De Vate, C. and Van Arkel, G.A. (1969) Mol. Gen. Genetics 105,152-163.

19. Dressler, D., Hourcade, D., Koths, K. and Sims, J. (1978) In Denhardt, D.T., Dressler, D. and Ray, D.S. (eds), The Single-stranded DNA Phages, Cold Spring Harbor Laboratory, Cold Spring Harbor, NY, pp. 187-214.

20. Baas, D.B. and Jansz, H.S. (1978) In Denhardt, D.T., Dressler, D. and Ray, D.S. (eds), The Single-stranded DNA Phages, Cold spring Harbor Laboratory, Cold spring Harbor, NY, pp. 215-244.

21. Lilley, D.M. and Kemper, B. (1984) Cell 36,413-422.

22. Mizuuchi, K., Kemper, B., Hays, J. and Weisberg, R.A. (1982) Cell 29,357-365.

23. Leach, D.R.F. and Stahl, F.W. (1983) Nature 305,448-451.

24. Warren, G.J. and Green, R.L. (1985) J. Bacteriol. 161,11031111 .

25. Collins, J. (1980) Cold spring Harbor symp. Quant. Biol. 45, 409-416.

26. Collins, J., Volckaert, G. and Nevers, P. (1982) Gene 19,139146 .

27. Weisbeek, P., Van Der Ende, A, Van Der Avoort, H., Teerstra, R., Van Mansfeld, F. and Langeveld, S. (1981) In Ray, D.S. (ed) The Initiation OF DNA Replication, Academic Press, Inc., New York, NY, pp.211-232.

28. Sanger, F., Coulson, A.R., Friedmann, T., Air, T., Barrell, B.G., Brown, N.L. Fiddes, J.C., Hutchinson III, C.A., Slocombe, P.M. and Smith, M. (1978). J. Mol. Biol. 125,225246 . 